\title{
HIV DNA and Dementia in Treatment-Naïve HIV-I-Iniected Individuals in Bangkok, Thailand
}

\section{Bruce Shiramizu1, Silvia Ratto-Kim ${ }^{12}$, Pasiri Sithinamsuwan ${ }^{3}$, Samart Nidhinandana 3 , Sataporn Thitivi- chianlert $^{3}$, George Watt ${ }^{1}$, Mark deSouza ${ }^{2}$, Thippawan Chuenchitra ${ }^{2}$, Suchitra Sukwit ${ }^{2}$, Suwicha Chitpatima ${ }^{4}$, Kevin Robertson ${ }^{5}$, Robert Paul6, Cecilia Shikuma ${ }^{1}$, Victor Valcour ${ }^{1}$}

\begin{abstract}
1. Hawaii AIDS Clinical Research Program, University of Hawaii, Honolulu, HI, USA; 2. Armed Forces Research Inst. Med. Sciences, Bangkok, Thailand; 3. Phramongkutklao Hosp., Bangkok, Thailand; 4. Royal Thai Army Med. Dept., Bangkok, Thailand; 5. Univ. North Carolina, Chapel Hill, NC, USA; 6. Univ. Missouri, Dept. Psychology, St. Louis, MO, USA - for the South East Asia Research Collaboration with the Univ. of Hawaii Protocol 001 Team.
\end{abstract}

Correspondence to: B. Shiramizu, MD; 3675 Kilauea Ave.; Young Bldg., 5th Floor; Honolulu, Hawaii, USA, 96816; Phone: 808-737-2751; Fax: 808-735-7047; bshirami@hawaii.edu

Received: 2006.11.16; Accepted: 2006.12.05; Published: 2006.12.06

High HIV-1 DNA (HIV DNA) levels in peripheral blood mononuclear cells (PBMC) correlate with HIV-1-associated dementia (HAD) in patients on highly active antiretroviral therapy (HAART). If this relationship also exists among HAART-naïve patients, then HIV DNA may be implicated in the pathogenesis of HAD. In this study, we evaluated the relationship between HIV DNA and cognition in subjects naïve to HAART in a neuroAIDS cohort in Bangkok, Thailand. Subjects with and without HAD were recruited and matched for age, gender, education, and CD4 cell count. PBMC and cellular subsets were analyzed for HIV DNA using real-time PCR. The median $\log _{10}$ HIV DNA copies per $10^{6}$ PBMC for subjects with HAD (n=15) was 4.27, which was higher than that found in subjects without dementia (ND; $n=15), 2.28, p<0.001$. This finding was unchanged in a multivariate model adjusting for plasma HIV-1 RNA levels. From a small subset of individuals, in which adequate number of cells were available, more HIV DNA was in monocytes/macrophages from those with HAD compared to those with ND. These results are consistent with a previous report among HAART-experienced subjects, thus further implicating HIV DNA in the pathogenesis of HAD.

Key words: human immunodeficiency virus type 1; dementia; cognition; HIV DNA

\section{INTRODUCTION}

Complete eradication of the human immunodeficiency virus, type 1 (HIV-1) from infected individuals is not currently possible due, in part, to continuing presence of virus in lymphocytes and cells of the macrophage lineage [1-3]. Monocytes/macrophages $(\mathrm{M} / \mathrm{M} \Phi)$ are cellular sanctuaries for HIV-1, which remain present even in patients with suppressed plasma viremia on highly active antiretroviral therapy (HAART) $[4,5]$. These cells may be particularly suited as sanctuaries for virus because HIV-1 DNA (HIV DNA), compared to HIV RNA, is less affected by current treatment regimens [6-9]. Additionally, these nondividing cells differ in many respects from that of CD4 lymphocytes making them unique entities for long-term persistence of HIV DNA [4, 10]. For instance, mitosis of $M / M \Phi$ is not required for nuclear import or integration of viral DNA; and M/M $\Phi$ not only contribute to establishment and persistence of HIV-1 infection, they also activate surrounding T-cells thus favoring their infection.

These circulating monocytes traffic through tissue and to the central nervous system (CNS) and differentiate into tissue macrophages. This provides a basis for theorizing that $\mathrm{M} / \mathrm{M} \Phi$ may contribute to the ongoing persistence of HIV-1 in these sites [11]. Monocyte trafficking to the CNS is hypothesized to be an underlying event in neuropathogenesis of HIV-1-associated dementia (HAD) [12-14]. Our recent observation of high HIV DNA levels in subjects with HAD, even among those with undetectable plasma HIV-1 RNA levels (VL), highlights the significance of PBMC HIV DNA in the pathogenesis of HAD [15]. We performed the prior study on subjects who were on HAART, therefore the question remains regarding the significance of HIV DNA in HAD pathogenesis before beginning therapy. In the prior study, our data suggested that HIV DNA was predominantly in CD14/CD16 M/MФ [15]. Therefore to further assess the importance of CD14/CD16 phenotype, the current study attempts to address the question whether a specific PBMC subset (M/MФ or CD4 lymphocytes) harbors HIV DNA.

We undertook the current study to test the hypothesis that HIV DNA levels would be elevated in cognitively-impaired individuals naïve to HAART; and that HIV DNA levels in M/MФ are higher than in CD4 lymphocytes in subjects with HAD compared to those with no dementia (ND). We hypothesize that an 
association of HIV DNA with HAD before starting HAART would further implicate HIV DNA in the neuropathogenesis of HAD likely due to the presence of virus in M/MФ that traffic to the CNS. The work was completed using a cohort in Bangkok, Thailand, which was established to characterize cognition among individuals initiating HAART for the first time as the country rapidly escalated access to antiretroviral drugs.

\section{METHODS}

\section{Subjects and Clinical Data.}

We established a longitudinal neuroAIDS cohort within the Southeast Asia Research Collaboration with the University of Hawaii (SEARCH) to characterize HIV-1-related cognitive dysfunction among individuals in Bangkok infected with the most commonly identified subtype in Thailand, recombinant circulating form (CRF) 01_AE. The protocol and consent forms were approved by the Ethical Review Committee and Institutional Review Board of the participating institutions. The SEARCH institutions involved in this project included the University of Hawaii, Phramongkutklao Hospital (PMK), and the Armed Forces Research Institute of Medical Sciences (AFRIMS), the latter two located on the same campus in Bangkok, Thailand. Study volunteers were enrolled at PMK, a large, tertiary care teaching hospital that is administered by the Royal Thai Army, which provides care for all Thai nationals regardless of military affiliation. The study enrolled Thai individuals living in Bangkok with HAD, ND, and HIV-1-seronegative controls matched for age, education, and gender. HIV-1-infected subjects were also matched for CD4 cell counts. The seronegative controls were enrolled and completed identical neuropsychological tests as the HIV-1-infected individuals because no Thai normative data were available to analyze the results. All individuals had minimal/distant or no exposure to illicit drug use with negative urine toxicology screens on two occasions within 30 days prior to enrollment. Subjects were all seronegative for hepatitis C virus, free of neurological or psychiatric illnesses including major depression, and did not have central nervous system opportunistic infection, active opportunistic infection in any organ system, pre-existing or known learning disability, or past brain trauma. Individuals thought to have cognitive impairment were referred for study participation from the outpatient neurology and infectious diseases clinics at PMK or from other hospitals/clinics. Matched HIV-1-infected individuals without HAD were then recruited.

The protocol neurologist (P. S.) established a diagnosis of HAD using standard-of-care approaches for Thailand. In general, the evaluation included participant and proxy informant reports of symptoms and function, the HIV macroneurological examination as used in the Adult AIDS Clinical Trails Group (AACTG, NIAID), bedside cognitive testing (including assessment of orientation, motor and psychomotor speed, memory, executive functioning, and visuospatial skill), and the international HIV-1 Dementia Scale [16]. All participants with HAD were further evaluated to rule-out other causes of cognitive impairment including gadolinium-enhanced brain MRI. If clinically indicated, individuals underwent a lumbar puncture to exclude opportunistic brain infection, however of the eight lumbar punctures that were performed, no opportunistic infections were found. Similarly, even though HIV-1-infected subjects had advanced immunosuppression, there were no individuals who had any history of any opportunistic infections, including in the CNS. After enrollment, all participants were evaluated with a modified version of the WHO International HIV-1 neuropsychological battery [17]. We selected this battery as it was designed to minimize cultural bias and was utilized in a prior study conducted in Bangkok; therefore feasibility was established [17]. We substituted the Brief Visual Memory Test-revised for the Picture Memory Test for logistical reasons as the latter required immediate and consistent computer and internet access. We assessed depressive symptoms with the Thai Depression Inventory (TDI) which was previously validated in Thailand [18]. The assessment was a clinical assessment made by the protocol neurologist (P. S.) at the time of clinical evaluation using the TDI, patient interview, and patient and proxy information to assist in this assessment.

We validated the diagnosis of HAD by reviewing the first 27 HIV-1 cases enrolled in a consensus panel consisting of an HIV neurologist, the study HIV neuropsychologist (R. P.) and the principal investigator of the cohort (V. V.). We prepared case summaries consisting of all clinical and neurological data. Individual raw neuropsychological scores were then plotted over 3 box plot distributions of seronegative controls, individuals with HAD, and those with ND. In a blinded fashion, we determined a consensus diagnosis of HAD or ND and reached consensus with the diagnosis determined by our Thai colleague on $100 \%$ of the ND cases. Among the HAD cases, the consensus panel was congruent in $70 \%$ of the cases with the remaining cases felt to be either mild dementia or minor cognitive motor disorder, with an overall congruence exceeding $85 \%$. The consensus panel was convened to validate the diagnosis by the Thai neurologist and not to substitute it. Therefore, since an excellent congruence was achieved, we completed the analysis using the original diagnoses for the purpose of this evaluation.

Viral load and CD4 lymphocyte counts were performed at AFRIMS, which maintains a Certificate of American Pathologists for these tests. Viral subtypes were determined by ELISA serotyping using V3-CM237 (Thai subtype B) and V3 CM242 (subtype E) peptides, which distinguishes HIV-1 subtype B and E infection in Thai individuals and confirmed by sequencing, when indicated [19, 20].

\section{Specimens and HIV DNA Assay.}

At entry into the cohort, PBMC were isolated and stored frozen in dimethyl sulfoxide from blood 
(ethylenediaminetetraacetic acid tube). DNA was extracted from an aliquot of frozen PBMC (5 X $10^{6}$ cells), as previously reported [15]. HIV DNA, normalized to the number of copies of HIV-1 DNA per $10^{6}$ cells, was then measured using real-time polymerase chain reaction (PCR), as previously described [15]. We performed all real-time PCR assays in triplicate using independent standard curves generated to measure relative HIV DNA copy number and cellular equivalent genomic DNA. The plasmid used to generate the standard curves was designed with a single copy each of HIV-1 and a housekeeping gene, $\beta$ globin. We used two primer sets to distinguish amplification of the two genes: HIV gag (conserved 296 base pair product for subtypes A and B) and Bglobin ( 330 base pair product). The PCR master mix consisted of either the HIV or $\beta$ globin primers and probe sets, $1 \mathrm{x}$ iQ supermix (BioRad Laboratories, Hercules, CA), $100 \mathrm{ng}$ DNA, and water (final volume $25 \mu \mathrm{L}$ ) with the following conditions: initial denaturation for $3 \mathrm{~min}$ followed by 45 cycles of $95^{\circ} \mathrm{C} / 10$ seconds, $57^{\circ} \mathrm{C} / 30$ seconds; with final extension of $72^{\circ} \mathrm{C} / 2 \mathrm{~min}$. We used non-HIV-1 infected genomic DNA for a negative control and DNA from three HIV-1 infected cell lines (8E5, OM10.1, and ACH-2; NIH AIDS Research and Reference Reagent Program, NIH, Bethesda, MD) as positive controls. The HIV-1 primers were tested on HIV-1 clades A, E, and $\mathrm{B}$; and demonstrated equivalent amplification of the target gene.

\section{Separation of PBMC Subsets and HIV DNA Analay- sis.}

Because HIV DNA is present in both lymphocytes and monocytes, we were interested in assessing whether more HIV DNA was in one particular PBMC subset versus the other. We previously measured HIV DNA in PBMC subsets from individuals from a different cohort and showed that there were higher levels in $\mathrm{M} / \mathrm{M} \Phi$ compared to CD14- cells in those diagnosed with HAD versus those with ND [15, 21]. The same procedures were performed on the specimens from HIV-1-positive subjects for the current study from which adequate numbers of cells were available to recover reasonable quantities of cells in the subsets. To separate the cells, we used RosetteSep (Stemcell Technologies, Vancouver, BC, Canada) combined with magnetic beads. Initially a CD14- subset from a small aliquot of blood, which includes CD4 lymphocytes, was isolated with beads; with the remaining cells separated into $\mathrm{CD} 14^{+} / \mathrm{CD} 16^{+}$by enrichment and bead separation. An aliquot of the sorted cells was then analyzed by flow cytometry (FACSCalibur, Becton Dickinson, San Jose, CA) to verify the phenotype in each subset. The cells were analyzed using FlowJo software (Tree Star Inc, San Jose, CA) following staining with the following antibodies (BD Biosciences, San Jose, CA): murine anti-human antibodies, FITC-conjugated anti-CD14, PE-conjugated anti-CD16 (3G8; PharMingen), PerCP-conjugated anti-HLA-DR, and isotype controls. Total DNA was isolated from each subset and HIV DNA measured as described above.

\section{Statistical Analysis.}

We used logistic regression models to examine the independent effect of HIV DNA on HAD vs. ND with the Likelihood-Ratio test on the odds-ratio. Analyses were conducted using SAS 9.0 (SAS Institute, Cary, N.C.) with a $p$-value $<0.05$ interpreted as a significant result. A two sample $t$ test for the education/age/CD4/VL variables and Fisher's Exact test for the gender variable were used.

Figure 1. HIV DNA in Subjects with HAD vs. Non-HAD. $\log _{10}$ HIV DNA levels in subjects with HAD ( $n=15$; median $=4.27)$ are higher than those without HAD (ND, $n=15$; median=2.28), $\mathrm{p}<0.001$.

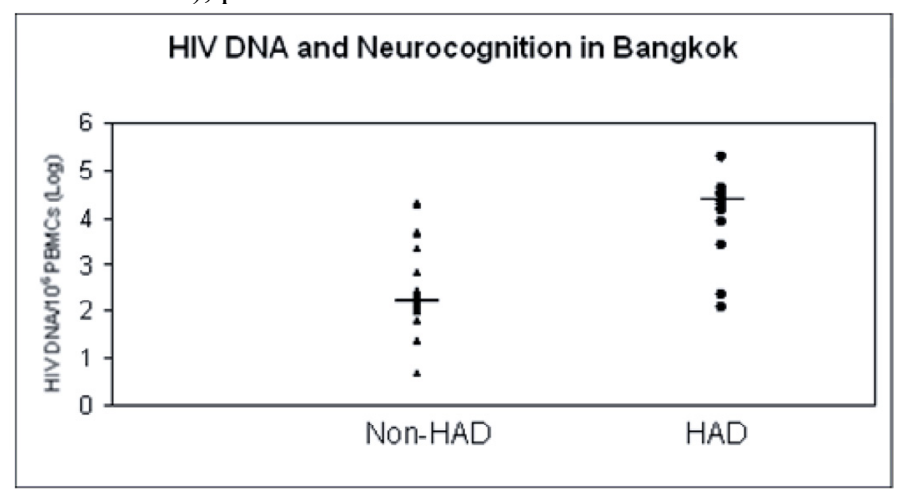

\section{RESULTS}

Sixty individuals entered the study $(\mathrm{n}=15$ each for the HAD and ND groups and $n=30$ for HIV-1 seronegative controls); matched for age, gender, and years of education, Table 1 . All participants were Thai nationals with the majority of the participants being female. The HIV-1-seropositive subjects $(n=30)$ were HAART-naïve initially and had relatively low CD4 cell counts prior to initiation of therapy, Table 1. All of the HIV-1-infected individuals were infected with circulating HIV-1 subtype (CRF) 01_AE. In subjects with HAD, compared to those with ND, the median (IQR) CD4 cell counts were 21 (6-74) cells/ $\mu \mathrm{L}$ and 39 (16-71) cells $/ \mu \mathrm{L}$, respectively, with no difference between the two groups, $p=0.775$. As would be expected, in treatment-naïve patients with low CD4 cell counts, the $\log _{10}$ HIV-1 plasma RNA levels were relatively high with no difference in between the two groups (median 5.28 and 5.33 for HAD and ND, respectively), $p=0.811$.

Comparing subjects with and without HAD, we found significantly higher $\log _{10}$ HIV DNA copies per $10^{6} \mathrm{PBMC}$ in the HAD group [ $\mathrm{n}=15$; median 4.27 (2.10 to 5.28)] versus the ND group [ $n=15 ; 2.28$ (0.69 to 4.30)], $p<0.001$, Table 1 . The calculated $\log _{10}$ HIV DNA $/ 10^{6}$ PBMC and medians for ND and HAD individuals is shown in Figure 1. In an unadjusted logistic regression model, we identified an association of HIV DNA to HAD resulting in an odds ratio of 1.841 (95\% confidence interval, CI, 1.286-2.635), $p<0.001$, with the odds ratio representing a one unit increase in $\log _{10}$ HIV DNA copies per $10^{6}$ cells. This effect was unchanged in a multivarate model adjusting for plasma 
HIV RNA levels (odds ratio 1.867, 95\% CI 1.297-2.688). HIV-1 seronegative control subjects.

As expected, HIV DNA was not detected in any of the

Table 1. Demographic and Laboratory Parameters

\begin{tabular}{|c|c|c|c|c|}
\hline \multicolumn{2}{|c|}{ HIV-1-Seronegative (n=30) } & HAD (n=15) & ND (n=15) & $p$ \\
\hline Age (years) [mean (SD)] & $34.1(9.6)$ & $33.1(8.6)$ & $33.7(8.0)$ & 0.947 \\
\hline Years of education [Mean (SD)] & $7.6(1.8)$ & $6.9(2.3)$ & $6.6(1.7)$ & 0.186 \\
\hline $\begin{array}{c}\text { Female/Male } \\
\text { CD4 cell count (cells/ } \mu \mathrm{L})\end{array}$ & $18 / 12$ & $9 / 6$ & $10 / 5$ & 0.904 \\
\hline $\begin{array}{c}\text { Median (IQR) } \\
\text { Log }_{10} \text { HIV-1 RNA (copies/mL) } \\
\text { Median (IQR) }\end{array}$ & $797.6(679-1012)$ & $21(6-74)$ & $39(16-71)$ & \\
\hline $\begin{array}{c}\log _{10} \text { HIV DNA (copies/106 PBMC) } \\
\text { Median (IQR) }\end{array}$ & Not applicable & $5.28(5.04-5.54)$ & $5.33(5.08$ to 5.53) & 0.811 \\
\hline
\end{tabular}

HAD: HIV-1-associated dementia; ND: no dementia

Table 2. HIV DNA Copy and Total Burden in PBMC and Subsets

\begin{tabular}{|c|c|c|c|c|c|c|c|}
\hline \multirow[t]{2}{*}{ Diagnosis } & \multicolumn{3}{|c|}{ HIV DNA Copy per } & \multicolumn{3}{|c|}{ Total HIV DNA Copy Calculated From } & \multirow[t]{2}{*}{ Ratio* $^{*}$} \\
\hline & PBMC & CD14/CD16 & CD4 & PBMC & CD14/CD16 & CD4 & \\
\hline HAD & $4.10 \times 10^{-2}$ & $1.27 \times 10^{-2}$ & $1.92 \times 10^{-4}$ & $2.50 \times 10^{8}$ & $1.48 \times 10^{6}$ & $3.5 \times 10^{4}$ & $>1$ \\
\hline HAD & $2.09 \times 10^{-2}$ & $2.16 \times 10^{-2}$ & $1.59 \times 10^{-4}$ & $1.23 \times 10^{8}$ & $3.15 \times 10^{6}$ & $9.38 \times 10^{3}$ & $>1$ \\
\hline HAD & $2.00 \times 10^{-2}$ & $1.24 \times 10^{-2}$ & $5.30 \times 10^{-4}$ & $1.08 \times 10^{8}$ & $1.79 \times 10^{6}$ & $5.72 \times 10^{4}$ & $>1$ \\
\hline Non-HAD & $1.89 \times 10^{-4}$ & $9.10 \times 10^{-6}$ & $1.56 \times 10^{-4}$ & $1.25 \times 10^{6}$ & $7.46 \times 10^{2}$ & $1.03 \times 10^{6}$ & $<1$ \\
\hline Non-HAD & $4.45 \times 10^{-3}$ & $3.77 \times 10^{-4}$ & $4.29 \times 10^{-3}$ & $2.83 \times 10^{7}$ & $2.70 \times 10^{4}$ & $1.62 \times 10^{6}$ & $<1$ \\
\hline Non-HAD & $4.68 \times 10^{-3}$ & $3.13 \times 10^{-4}$ & $4.08 \times 10^{-3}$ & $1.54 \times 10^{7}$ & $2.41 \times 10^{4}$ & $5.39 \times 10^{5}$ & $<1$ \\
\hline Non-HAD & $2.00 \times 10^{-2}$ & $1.06 \times 10^{-3}$ & $1.94 \times 10^{-2}$ & $1.10 \times 10^{8}$ & $6.76 \times 10^{4}$ & $6.40 \times 10^{6}$ & $<1$ \\
\hline
\end{tabular}

*Ratio of CD14/CD16 to CD4 > 1.00 denotes total higher HIV DNA levels in CD14/CD16 compared to CD4 subsets

Figure 2. Phenotypic Expression of $\mathrm{CD} 14^{+}$and $\mathrm{CD} 14^{-}$Sorted Subsets. Cells from sorted fractions were stained for CD14. A, B) Two examples of CD14-stained sorted cell populations from monocyte fractions from two different subjects demonstrating the majority of cells isolated were $\mathrm{CD}_{1} 4^{+}(82.3 \%$ and $92.3 \%)$; C) CD14-negative subset showing low CD14-staining $(0.49 \%)$.

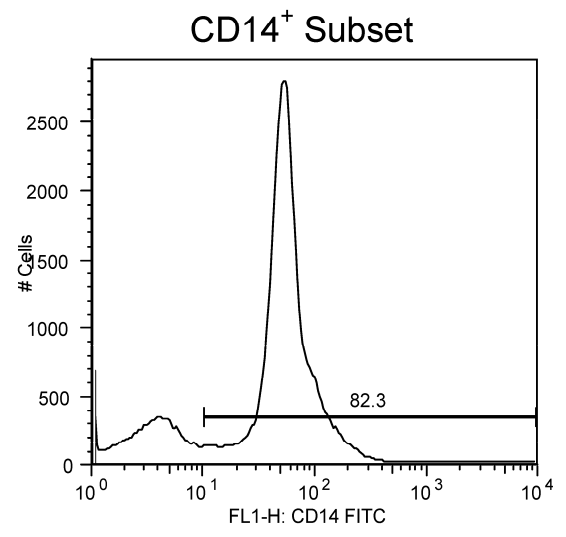

A

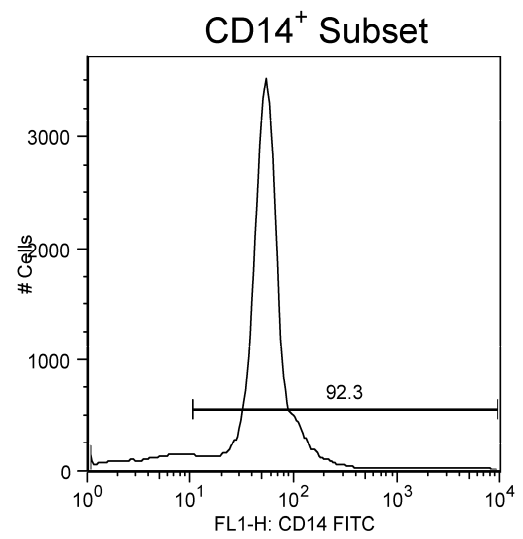

$\mathrm{B}$

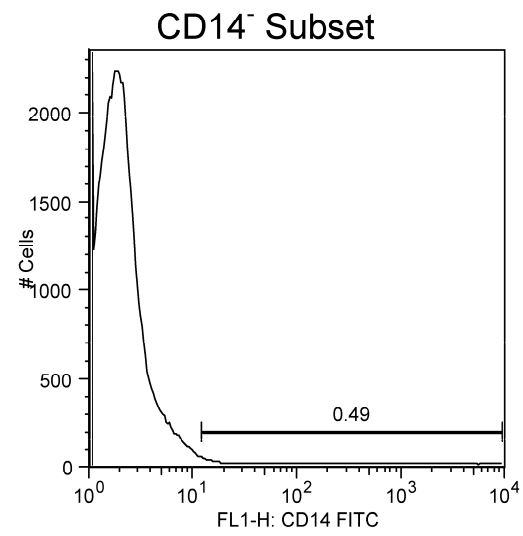

C
A limited number of individuals (HAD $n=5$; ND $\mathrm{n}=4$ ) had analyses of PBMC subsets in which an adequate number of cells was available for separation. Using flow cytometry (monocyte \& CD4/CD8 percentages) and data from sorted cells, we estimated the total HIV DNA copies from CD14/CD16 and CD4 subsets. An assumption was made whereby HIV DNA measurements from the CD14- subsets were primarily from CD4 lymphocytes. The efficiency of our sorting procedure is depicted in Figures $2 \mathrm{~A} \& 2 \mathrm{~B}$, where greater than $80-90 \%$ of isolated monocyte subsets were 
CD14 ${ }^{+}$. Less than $1 \%$ of cells from the CD14- subset were positive for CD14, Figure 2C. By extrapolating from the calculated values, HIV DNA levels in PBMC were relatively high in all individuals diagnosed with HAD with the highest in the CD14/CD16 subsets compared to CD4 subsets, Table 2. We initially estimated HIV DNA copy per PBMC; per CD14/CD16 cell; and per CD4 lymphocyte, Table 2 . We then estimated the total HIV DNA contribution from each subset using the flow data and complete blood counts obtained at the same time the blood was collected. In this analysis, the total HIV DNA contribution from CD14/CD16 cells was higher than the HIV DNA contribution from CD4 cells in subjects with HAD, Table 2, which was not apparent in subjects without HAD. In order to estimate differences in the HIV DNA contribution from the two subsets, the ratios of HIV DNA from CD14/CD16 subsets to HIV DNA from CD4 subsets were calculated. This resulted in ratios significantly higher from those with $\operatorname{HAD}(n=5$; median=188.5) compared to those with ND ( $n=4$; median=0.0059), $\mathrm{p}<0.029$, Table 2 .

\section{DISCUSSION}

Current antiretroviral therapy for HIV-1 focuses on eradication of the virus from plasma. In contrast to the cytotoxic effects of HIV-1 on lymphocytes, HIV-1-infection usually leads to chronic infection in M/MФ. Recent studies suggest that PBMC HIV DNA may be a marker for HIV-1 disease progression [22-25]. Our laboratory previously reported the presence of high HIV DNA in PBMC as a risk for HAD in HAART-experienced individuals; and preliminary analyses suggest that the majority of this HIV DNA may be in circulating $\mathrm{M} / \mathrm{M} \Phi$ [15]. We demonstrated that this effect was independent of plasma HIV-1 RNA levels by a separate analysis of HIV DNA in individuals with undetectable plasma VL. We now confirm our findings in a different cohort who are naïve to HAART and hypothesize that high HIV DNA levels are an important factor in HAD pathogenesis.

In the current study, we found the effect of HIV DNA on HAD was independent of age and current CD4 count at the time of recruitment, which is similar to what was found previously in patients on effective antiretroviral therapy. The HIV DNA data suggesting a higher contribution from the monocyte/macrophage subsets in patients with HAD are limited by the small number of specimens available. The cohort established in Thailand provided a unique opportunity to test our hypothesis of the role of HIV DNA in HAD. We were able to enroll age-, education-, and gender-match HIV-1 seronegative individuals as controls to establish normative data for the current study, which have not previously been established in Thailand. Additional data in PBMC subsets are needed to assess the importance of HIV DNA in the pathogenesis of HAD. Other limitations of the current study include the assumption that the CD14- subsets were composed mainly of CD4 lymphocytes. The calculations of HIV DNA copies in the PBMC subsets are based on extrapolated values. To confirm the findings, future experiments are planned to use a cell sorter to isolate specific cell populations.

While the mechanism by which HIV DNA leads to neurocognitive problems remains unclear, we propose that our results demonstrating an association in HAART-naïve patients strengthens the relationship of HIV DNA to HAD neuropathogenesis. Even though the mechanisms linking HIV DNA to HAD pathogenesis are not fully known, studying HIV DNA in PBMC subsets such as memory and naïve CD4 T-lymphocytes, and $\mathrm{CD} 14^{+}$monocytes may provide clues to HIV-1-associated neuropathogenesis [6]. Others have shown that HIV DNA was detected in both T lymphocytes and monocytes in severely immunocompromised subjects on HAART, but with higher levels in monocytes [26]. In another study, monocytes were identified as the predominant cellular reservoir of virus in the majority of subjects who had been on HAART for longer than 2 years [24]. Calcaterra et al. found higher levels of HIV DNA in monocytes than in $\mathrm{CD}^{+}$lymphocytes in a subset of non-viremic patients [24]. Pertinent to our results was the finding that three patients in the Calcaterra analyses had HIV DNA titers in monocytes that were at least six-fold higher than in CD4+ lymphocytes [24].

Activated $\mathrm{CD}^{+}$lymphocytes, once infected, are rapidly killed by HIV-1 while M/M $\Phi$ are less affected by the cytopathic effect of the virus [27-29]. Several studies demonstrated the presence of HIV-1 in M/MФ in HAART-treated patients, even among those with consistently undetectable viral loads [15, 30-32]. The presence of elevated HIV DNA levels in PBMC in HAART-naïve and HAART-treated individuals with HAD relative to ND suggests a critical need to identify the interrelationship among $\mathrm{M} / \mathrm{M} \Phi, \mathrm{HIV}$ DNA, and HAD. This may expose underlying mechanisms to explain the continued prevalence of HAD in the era of HAART. Since HIV DNA in M/MФ persists while individuals are on HAART and since monocytes likely play a critical role in HIV-1 neuropathogenesis, these M/MФ may be important cellular reservoirs of virus $[33,34]$. Future studies are planned to assess other markers of monocyte/macrophage activation other than CD14/CD16 to determine the importance of HIV DNA in M/MФ in the pathogenesis of HAD.

In summary, our findings confirm the association between HIV DNA and dementia in HIV-1-infected patients even prior to instituting HAART. We also demonstrate that this effect does not appear to relate to age, CD4 count, or plasma HIV-1 RNA levels. The current study provides new evidence supporting the hypothesis that HIV DNA may be an important factor in HIV-1 neuropathogenesis. Further research is necessary to understand the mechanisms underlying this relationship, and particularly, to evaluate longitudinal cohorts to determine the prognostic significance of HIV DNA and its relationship to HAD incidence.

\section{ACKNOWLEDGEMENTS}

The work was presented, in part, at the 13th 
Conference on Retroviruses and Opportunistic Infections in Denver, CO, Feb. 4-7 2006. The work was support by National Institutes of Health Grants MH072388, U54NS43049, G12RR03061, MH69173, and U01A134853. The authors would like to thank Drs. Justin McArthur, Siripan Phatisawad, and Rapee Trichavaroj; Sean Hill, David Troelstrup, Erik Anderson, Andrew Williams, and Wichitra Apateerapong; and as well as acknowledge the SEARCH participants for their support and commitment.

\section{CONFLICT OF INTERESTS}

The authors declare no conflict of interests.

\section{REFERENCES}

1. Collman RG, Perno CF, Crowe SM, Stevenson M, and Montaner LJ. HIV and cells of macrophage/dendritic lineage and other non-T cell reservoirs: new answers yield new questions. J Leukoc Biol. 2003; 74: 631-634.

2. Aquaro S, Bagnarelli P, Guenci T, et al. Long-term survival and virus production in human primary macrophages infected by human immunodeficiency virus. J Med Virol. 2002; 68: 479-488.

3. Wong JK, Hezareh M, Gunthard HF, et al. Recovery of replication-competent HIV despite prolonged suppression of plasma viremia. Science. 1997; 278: 1291-1295.

4. Crowe S, Zhu T, and Muller WA. The contribution of monocyte infection and trafficking to viral persistence, and maintenance of the viral reservoir in HIV infection. J Leukoc Biol. 2003; 74: 635-641.

5. Siliciano RF. Scientific rationale for antiretroviral therapy in 2005 : viral reservoirs and resistance evolution. Top HIV Med. 2005; 13: 96-100.

6. Delobel P, Sandres-Saune K, Cazabat M, et al. Persistence of distinct HIV-1 populations in blood monocytes and naive and memory CD4 T cells during prolonged suppressive HAART. AIDS. 2005; 19: 1739-1750.

7. Crowe SM and Sonza S. HIV-1 can be recovered from a variety of cells including peripheral blood monocytes of patients receiving highly active antiretroviral therapy: a further obstacle to eradication. J Leukoc Biol. 2000; 68: 345-350.

8. Bagasra $\mathrm{O}$ and Pomerantz RJ. Human immunodeficiency virus type I provirus is demonstrated in peripheral blood monocytes in vivo: a study utilizing an in situ polymerase chain reaction. AIDS Res Hum Retroviruses. 1993; 9: 69-76.

9. Innocenti $\mathrm{P}$, Ottmann $\mathrm{M}$, Morand $\mathrm{P}$, Leclercq $\mathrm{P}$, and Seigneurin JM. HIV-1 in blood monocytes: frequency of detection of proviral DNA using PCR and comparison with the total CD4 count. AIDS Res Hum Retroviruses. 1992; 8: 261-268.

10. Verani A, Gras G, and Pancino G. Macrophages and HIV-1: dangerous liaisons. Mol Immunol. 2005; 42: 195-212.

11. Sonza S, Maerz A, Deacon N, et al. Human immunodeficiency virus type 1 replication is blocked prior to reverse transcription and integration in freshly isolated peripheral blood monocytes. J Virol. 1996; 70: 3863-3869.

12. González-Scarano F and Martín-García J. The neuropathogenesis of AIDS. Nature Reviews Immunology. 2005; 5: 69-81.

13. Gartner S. HIV infection and dementia. Science. 2000; 287 : 602-604.

14. Gendelman HE, Lipton SA, Tardieu M, Bukrinsky MI, and Nottet HS. The neuropathogenesis of HIV-1 infection. J Leukoc Biol. 1994; 56: 389-398.

15. Shiramizu B, Gartner S, Williams A, et al. Circulating proviral HIV DNA and HIV-associated dementia. AIDS. 2005; 19: 45-52.

16. Sacktor NC, Wong M, Nakasujja N, et al. The International HIV Dementia Scale: a new rapid screening test for HIV dementia. AIDS. 2005; 19: 1367-1374.
17. Maj M, Janssen R, Starace F, et al. WHO Neuropsychiatric AIDS study, cross-sectional phase I. Study design and psychiatric findings. Arch Gen Psychiatry. 1994; 51: 39-49.

18. Lotrakul M and Sukanich P. Development of the Thai Depression Inventory. J Med Assoc Thai. 1999; 82: 1200-1207.

19. Gaywee J, Artenstein AW, VanCott TC, et al. Correlation of genetic and serologic approaches to HIV-1 subtyping in Thailand. J Acquir Immune Defic Syndr Hum Retrovirol. 1996; 13: 392-396.

20. Mason CJ, Kitsiripornchai S, Markowitz LE, et al. Nationwide surveillance of HIV-1 prevalence and subtype in young Thai men. J Acquir Immune Defic Syndr Hum Retrovirol. 1998; 19: 165-173.

21. Shiramizu B, Williams A, Ratto-Kim S, et al. Activated Macrophages as a Potential Source of HIV DNA in HIV-1-Associated Dementia. In: 6th International Workshop on HIV, Cells of Macrophage/Dendritic Lineage, and Other Reservoirs. 2005. Verenna, Italy.

22. Viard JP, Burgard M, Hubert JB, et al. Impact of 5 years of maximally successful highly active antiretroviral therapy on CD4 cell count and HIV-1 DNA level. AIDS. 2004; 18: 45-49.

23. Havlir DV, Koelsch KK, Strain MC, et al. Predictors of residual viremia in HIV-infected patients successfully treated with efavirenz and lamivudine plus either tenofovir or stavudine. J Infect Dis. 2005; 191: 1164-1168.

24. Calcaterra S, Cappiello G, Di Caro A, Garbuglia AR, and Benedetto A. Comparative analysis of total and integrated HIV-1 DNA in peripheral CD4 lymphocytes and monocytes after long treatment with HAART. J Infect. 2001; 43: 239-245.

25. Rouzioux C, Hubert JB, Burgard M, et al. Early Levels of HIV-1 DNA in Peripheral Blood Mononuclear Cells Are Predictive of Disease Progression Independently of HIV-1 RNA Levels and CD4+ T Cell Counts. J Infect Dis. 2005; 192: 46-55.

26. Garbuglia AR, Zaccarelli M, Calcaterra S, et al. Dynamics of viral load in plasma and HIV DNA in lymphocytes during highly active antiretroviral therapy (HAART): high viral burden in macrophages after 1 year of treatment. J Chemother. 2001; 13: 188-194.

27. Perelson AS, Essunger P, Cao Y, et al. Decay characteristics of HIV-1-infected compartments during combination therapy. Nature. 1997; 387: 188-191.

28. Garaci E, Palamara AT, Ciriolo MR, et al. Intracellular GSH content and HIV replication in human macrophages. J Leukoc Biol. 1997; 62: 54-59.

29. Gendelman HE, Orenstein JM, Martin MA, et al. Efficient isolation and propagation of human immunodeficiency virus on recombinant colony-stimulating factor 1-treated monocytes. J Exp Med. 1988; 167: 1428-1441.

30. Belmonte L, Bare P, Picchio GR, et al. Decreased recovery of replication-competent HIV-1 from peripheral blood mononuclear cell-derived monocyte/macrophages of HIV-positive patients after 3 years on highly active antiretroviral therapy. AIDS. 2002; 16: 1289-1292.

31. Sonza S, Mutimer HP, Oelrichs R, et al. Monocytes harbour replication-competent, non-latent HIV-1 in patients on highly active antiretroviral therapy. AIDS. 2001; 15: 17-22.

32. Zhu T, Muthui D, Holte S, et al. Evidence for human immunodeficiency virus type 1 replication in vivo in CD14(+) monocytes and its potential role as a source of virus in patients on highly active antiretroviral therapy. J Virol. 2002; 76: 707-716.

33. Xiong $\mathrm{H}$, Zeng YC, Lewis $\mathrm{T}$, et al. HIV-1 infected mononuclear phagocyte secretory products affect neuronal physiology leading to cellular demise: relevance for HIV-1-associated dementia. J Neurovirol. 2000; 6 (Suppl 1): S14-23.

34. Kedzierska $\mathrm{K}$ and Crowe SM. The role of monocytes and macrophages in the pathogenesis of HIV-1 infection. Curr Med Chem. 2002; 9: 1893-1903. 